\title{
KEY HUMAN FACTORS AND THEIR EFFECTS ON HUMAN CENTERED ASSEMBLY PERFORMANCE
}

\author{
Maji I. Abubakar \\ University of Portsmouth, School of Mechanical and Design Engineering, Portsmouth, UK \\ maji.abubakar@myport.ac.uk \\ Qian Wang \\ University of Portsmouth, School of Mechanical and Design Engineering, Portsmouth, UK \\ qian.wang@port.ac.uk
}

\begin{abstract}
-
Today in the increasingly competitive market, consumers prefer to have a great variety of products to choose from; this preference is often coupled with demands for a relatively smaller lot size, shorter lead time, higher quality and lower cost. Consequently, manufacturing companies are being forced to consistently increase flexibility and responsiveness of their production systems in order to accommodate changes of the fluctuating market. Among various forms of production systems, human-centred manufacturing systems can offer such a capability in dealing with product variations and production volumes as human workers can always adapt themselves to perform multiple tasks after a learning process. However, human performance can also be unpredictable and it may alter due to varying psychological and physiological states, which are often overlooked by researchers when designing, implementing or evaluating a manufacturing system. This paper presents a study aiming to address these issues by exploring human factors and their interactions that may affect human performance on human-centred assembly systems. The study was carried out based on a literature review and an industrial survey. Critical system performance indicators, which are affected by human factors, were evaluated and the most significant human factors were identified using the fuzzy extent analysis method. The research findings show that experience is the most significant human factor that affects individual human performance, compared to age and general cognitive abilities in humancentred assembly. By contrast, both human reaction time and job satisfaction have the least effect on human performance. The significance of ageing on human performance was also studied and it was concluded that average assembly time of human workers rises by average $1 \%$ per year after the age of 38 years old.
\end{abstract}

Keywords: manufacturing systems, human factors, assembly, fuzzy extent analysis, ageing 


\section{Introduction}

In the past decade, most industrial companies have been shifting their manufacturing activities from mass production to mass customization aiming to increase product varieties and production volumes with small lot sizes, short lead times, high quality and low cost. One form of production systems is human-centred assembly systems, which can deal with variations in term of product mix and production volume as human workers can always adapt to production changes with varying demands from the competitive market. However, human capacity or performance in production is often affected by a variety of human factors interacting in a complex way (Schmid 2005). Nevertheless, such a phenomenon is often under or overestimated or simply neglected in manufacturing systems design, evaluation and implementation (Boenzi et al. 2015; Digiesi 2006; Baines et al. 2004).

Most studies have focused on the impact of human factors on human performance in general terms, which are not specifically related to manufacturing activities or production systems. Govindaraju et al. (2001) investigated the relations among ergonomic work conditions, human performance and quality based on a number of case studies. Boenzi et al. (2015) examined the variation of human performance between older workers and younger workers and concluded that this was insignificant. Giniger et al. (1983) observed that the effects of age and physiological fuctions were not significant, and both cognitive and physiological decline can be compensated by experience. By contrast, Hunter (1986) argued that some cognitive abilities may decline with age, while others may stablise over the life cycle, although fluid abilities (such as reasoning and working memory) can decline over age. However, crystallised abilities, which depend on accumulated knowledge, tend to remain stable (Zwick \& Gobel 2009; Deary et al. 2001; Warr 1994). Hunter et al. (1996) observed that a higher human performance can be attained by people who learn and transfer their skills to new tasks, although varying levels of individual performance may depend on their individual learning rates. Hunter (1986) concluded that it is the general cognitive ability that may determine human performance as it controls human capability with how much and how quick a person can learn.

A study by Belbase \& Sanzenbacher (2016) indicated that even workers with less ability to process information may also maintain productivity with the advancing age. Zwick et al. (2009) observed that the average muscle strength of a human decreases by roughly $10 \%$ per decade from 20 to 60 years old, 15\% from 60 to 80 years old and $30 \%$ after 80 years old. This 
may be due to aerobic capacity that reaches its peak at ages of 20 s and after these ages it declines by around $1 \%$ per year. Shephard (2000) reported that age affects the occupational performance of older individuals due to their decline in aerobic power. Moreover, Wang et al. (2012) stated that some costs in production may incure due to learning and forgetting of human operators who offer flexibility and responsiveness of a manufacturing system. Reagans et al. (2005) examined the relationship between worker experience and human learning and forgetting; it was observed that effect of forgetting was not significant when dealing with relatively less complex tasks.

A study shows that human reaction time tends to be fastest at the age of 20 years old; after this age it declines slowly until the age of 60 years old. It declines much faster after age of 70 years old and onwards. The study also shows that the reaction times of females are more volatile, compared to males (Deary et al. 2001). Doroudgar et al. (2017) used a simple visual reaction test to measure reaction times between a group of younger adult drivers (age from 18 to 40 years old) and a group of older adult drivers (60 years old and above), the experiemental result shows that the group of older drivers had the significantly poorer performance in reaction time leading to slower driving speed and more accidents. Adam et al. (1999) investigated the relationship between general cognitive ability and reaction time and concluded that there is a correlation between intelligence and reaction time, which, for males, is faster than females in almost all aging groups. However, Berg et al. (2006) stated that human reaction time can be affected by other issues of such as distraction and mental fatigue.

This paper reports an investigation of human factors and their interactions that may affect human performance on human-centred assembly systems; the work was carried out based on a literature study and an industrial survey. Critical system performance indicators, which are affected by human factors, were also evaluated, and the most significant human factors were identified using the fuzzy extent analysis approach.

\section{Review of previous studies}

A study by Broadbent (1971) indicated that human activity, which requires visual alertness, may be affected by sound, which distracts information intake and analysis. Avolio et al. (1990) used the polynomial regression analysis to predict work performance in connection with age and experience; the research outcome indicates that experience rather than age determines human performance. Schmidt et al. (1986) developed a model using a path analysis approach to examine the underlying influence of worker experience and job 
knowledge. The research result suggested that worker experience is the most influencial factor affecting human performance. The study by McDaniel et al. (1988) indicated that there is a direct relationship between job experience and job performance regardless of job complexity. Llmarinen (2001) observed that age may negatively affect general cognitive abilities but positively affect experience of a human worker, although experience may positively affect cognitive skills, which directly affect job peformance. Llmarinen (2001) also stated that physiological ability may decline due to aging; but it can also be compensated by experience gained as the age increases. Despite the decline of both cognitive and physical functions of a human worker due to aging, Giniger et al. (1983) and Stead et al. (1983) argued that the influence is not significant for older workers who may attain satisfactory job performance by applying cautions and restraints. Zwick et al. (2009) stated that human performance may be affected more by experience than aging. Kenny et al. (2015) investigated the physiological effect on decline of aerobic and musculoskeletal capacity due to aging; the study shows an average drop of $20 \%$ of physical work capacity at ages from 40 to 60 years old. A study by Avolio et al. (1990) shows experience rather than age of older workers is the key factor that is used for determining human performance as experience may offer an equal or even higher performance in comparison with their younger counterparts. In particlar the effect of experience appears to be more significant when performing a complex task. Rhodes (1983) suggested that human performance is more affected by age and Waldman et al. (1986) challenged some of Rhodes's conclusions arguing that the method used for the study may lead to unclear or even wrong interpretations. Furthermore, a study by McEvoy et al. (1989) showed that there is no clear relationship between age and job performance. Waldman et al. (1986) suggested that the effects of age and experience on performance may be subject to the cognitive demand of a task. Stanley (1985) investigated the influence of age on productivity of individuals and concluded that the effect of age on job performance may depend on the complexity of a task as complexity requires a strong mental capability that may deteriorate with ageing. A study by Skirbekk (2008) showed that the performance of individuals may differ for many reasons; this includes length of work experience, cognitive abilities, physical abilities and other relevant factors (such as environmental factors). Table 1 provides a summary of the effects of human factors on human performance for production, the results were obtained based on a literature review.

Table 1 Effect of human factors on human performance for production

Effects from $\quad$ Effects on $\quad$ Authors




\begin{tabular}{|c|c|c|}
\hline Physical work capacity & Cycle time & $\begin{array}{l}\text { Galen (1987); Govindaraju et al. (2001); Boenzi et } \\
\text { al. (2015) }\end{array}$ \\
\hline & Job satisfaction & $\begin{array}{l}\text { Govindaraju et al. (2001); Narahari \& Koneru } \\
\text { (2017) }\end{array}$ \\
\hline \multirow[t]{8}{*}{ Age } & $\begin{array}{l}\text { Physical work } \\
\text { capacity }\end{array}$ & $\begin{array}{l}\text { Shepherd (1999); Schibye \& Hansen (2001); Bridger } \\
\text { (2009); Stead \& page (1983); Zwick \& Gobel } \\
\text { (2009); Kenny et al. (2015). }\end{array}$ \\
\hline & Cycle time & N/A \\
\hline & Reaction time & $\begin{array}{l}\text { Woodson et al. (1992); Der \& Deary (2006); Salvia } \\
\text { et al. (2016), Svetina (2016); Doroudgar et al. (2017) }\end{array}$ \\
\hline & Idle time & N/A \\
\hline & $\begin{array}{l}\text { Learning and } \\
\text { forgetting }\end{array}$ & $\begin{array}{l}\text { Zwick \& Gobel (2009); Stanley (1985); Boenzi et } \\
\text { al. (2015) }\end{array}$ \\
\hline & Throughput & Baines, et al (2004) \\
\hline & $\begin{array}{l}\text { General Cognitive } \\
\text { Abilities }\end{array}$ & $\begin{array}{l}\text { Warr (1994); Boenzi et al. (2015); Stanley (1985); } \\
\text { Llmarinen (2001) }\end{array}$ \\
\hline & Job satisfaction & $\begin{array}{l}\text { Rhodes (1983); Drabe et al. (2015); Clark \& Oswald } \\
\text { (1996); Kumar (2017) }\end{array}$ \\
\hline \multirow[t]{2}{*}{ Gender } & $\begin{array}{l}\text { Physical work } \\
\text { capacity }\end{array}$ & Bridger (2009) \\
\hline & Reaction time & $\begin{array}{l}\text { Woodson (1992); Der \& Deary (2006); Adam et al. } \\
\text { (1999) }\end{array}$ \\
\hline \multirow[t]{8}{*}{ Experience } & Cycle time & Reagans et al. (2005) \\
\hline & Throughput & $\begin{array}{l}\text { Reagans et al. } \\
\text { (2005); Hunter (1986) }\end{array}$ \\
\hline & $\begin{array}{l}\text { Learning and } \\
\text { forgetting }\end{array}$ & $\begin{array}{l}\text { Shafer et al. (2001); Hunter \& Schmidt (1996); } \\
\text { Reagans et al. (2005) }\end{array}$ \\
\hline & $\begin{array}{l}\text { Physical work } \\
\text { capacity }\end{array}$ & Giniger et al. (1983) \\
\hline & Reaction time & Ando et al. (2004); Visser et al. (2007) \\
\hline & $\begin{array}{l}\text { General Cognitive } \\
\text { Abilities }\end{array}$ & $\begin{array}{l}\text { Giniger et al. (1983); Llmarinen (2001); Boenzi et al. } \\
\text { (2015) }\end{array}$ \\
\hline & $\begin{array}{l}\text { In-process } \\
\text { inventory }\end{array}$ & N/A \\
\hline & Idle time & N/A \\
\hline \multirow[t]{4}{*}{$\begin{array}{l}\text { Learning and } \\
\text { forgetting }\end{array}$} & Cycle time & $\begin{array}{l}\text { Nembhard \& Osothsilp (2002); Falck \& Rosenqvist } \\
\text { (2012a) }\end{array}$ \\
\hline & Throughput & $\begin{array}{l}\text { Shafer et al. (2001); Nembhard \& Osothsilp (2002); } \\
\text { Hunter (1986); Wang et al. (2012); Digiesi et al. } \\
\text { (2006) }\end{array}$ \\
\hline & $\begin{array}{l}\text { In process } \\
\text { inventory }\end{array}$ & N/A \\
\hline & Idle time & N/A \\
\hline \multirow[t]{3}{*}{ Job satisfaction } & Throughput & Rodriguez et al. (2016) \\
\hline & Throughput & Bridger (2009); Bainess \& Kay (2002) \\
\hline & Reaction time & Welford (1968); Moskaliuk et al. (2017) \\
\hline \multirow{3}{*}{$\begin{array}{l}\text { General Cognitive } \\
\text { Abilities }\end{array}$} & Reaction time & Deary et al. (2001), Govindaraju et al. (2001) \\
\hline & $\begin{array}{l}\text { Learning and } \\
\text { forgetting }\end{array}$ & Hunter (1986); Wirojanagud et al. (2007) \\
\hline & Throughput & Hunter \& Schmidt (1996); Wirojanagud et al. (2007) \\
\hline
\end{tabular}




\begin{tabular}{lll}
\hline & Cycle time & Govindaraju et al. (2001) \\
\hline & Idle time & N/A \\
\hline Circadian rhythms & Reaction time & Berg \& Neely (2006) \\
\hline
\end{tabular}

In this work, an industrial survey was also conducted to compare the findings with the corresponding outcomes obtained based on the literature study. This process was involved in testing and selecting 33 effective respondents, of whom, $60 \%$ were researchers in the field of engineering, $30 \%$ were industrialists and $10 \%$ were from other sectors. The relationship between identified human factors on human-centred performance were rated using the Likert scale (Allen \& Seaman 2007). Respondents were asked to rate the influence of one human factor over another using a five-point scale; it gave a mean of 3.0 based on scales rated by respondents to all the questions. Table 2 shows the calculated mean value of the cumulative responses for each question using a statistical package for social science (SPSS). In this study, any value obtained below 3.0 was considered as a weak relationship and these values were filtered out. The mean values, as shown in Table 2, were assigned to the linguistic terms depicting the amplitude of effects between human factors. As an example, a mean value of 3.54 indicates a relatively weaker impact of ageing on learning and forgetting. Further, these mean values are categorised into four classes as shown in Table 3.

Table 2 Measurement of the effective-relationships between human factors

\begin{tabular}{lcc}
\hline Human factor & Mean & Std dev. \\
\hline Experience (EX) vs Age (AG) & 4.48 & 0.50 \\
\hline Experience (EX) vs Reaction Time (RT) & 4.27 & 0.67 \\
\hline General Cognitive Abilities (GCA) vs Learning and Forgetting (LF) & 4.27 & 1.03 \\
\hline Experience (EX) vs Reaction Time (RT) & 4.27 & 0.67 \\
\hline Experience (EX) vs Physical Work Capacity (PW) & 4.24 & 0.86 \\
\hline Age (AG) vs Physical Work Capacity (PW) & 4.15 & 1.00 \\
\hline Experience (EX) vs Learning and Forgetting (LF) & 4.06 & 0.86 \\
\hline Age (AG) vs Job satisfaction (JS) & 4.06 & 1.14 \\
\hline Age (AG) vs General Cognitive Abilities (GC) & 4.00 & 0.75 \\
\hline General Cognitive Abilities (GC) vs Reaction Time (RT) & 3.93 & 0.86 \\
\hline Physical Work Capacity (PW) vs Job satisfaction (JS) & 3.93 & 1.27 \\
\hline Age (AG) vs Reaction Time (RT) & 3.81 & 1.01 \\
\hline
\end{tabular}




\begin{tabular}{lcc}
\hline Age (AG) vs Learning and Forgetting (L,F) & 3.54 & 1.25 \\
\hline Gender (GD) vs Reaction Time (RT) & 2.87 & 0.89 \\
\hline Circadian Rhythms (CR) vs Reaction Time (RT) & 2.87 & 0.85 \\
\hline Gender (GD) vs Physical Work Capacity (PW) & 2.72 & 0.94 \\
\hline
\end{tabular}

\section{Analytical hierarchy process and fuzzy set theory}

Human behaviours can be difficult to measure; their interdependence or relationships are often ambiguous and still not well understood. Descriptions of human behaviour or performance are generally linguistic (Karwowski \& Mital 1986). Therefore, a multi-criteria decision making tool, namely the analytical hierarchy process (AHP), was used for selecting the solution based on the subjective judgements. AHP, however, is criticised for disregarding the vagueness and prejudice of human judgements, i.e., it does not account for human thinking, especially as it relates to human attributes or human traits, which may not be easily evaluated using conventional numbers, apart from the language expression (Tzeng \& Huang 2011; Saaty 2008; Aggarwal \& Singh 2013). Zadeh (1965) developed a methodology using fuzzy sets as a way in which sharp numerical values can be represented using overlapping boundaries of fuzzy numbers taking into account inherent human imprecision in a decision making process (Mikhailov 2003; Fan et al. 2004; Chen 2001; Chou et al. 2008; Hwang \& Yoon 2004). Fuzzy set theories are introduced and defined in the literature by Buckley (1985) and Laarhoven \& Pedrycz (1983). The concept of fuzzy AHP was developed by embedding the standard AHP approach into a fuzzy domain; the earlier study on the fuzzy AHP approach was made by Laarhoven \& Pedrycz (1983). The triangular fuzzy numbers (TFNs) are widely used in the fuzzy analytical hierarchy process (FAHP) (Laarhoven \& Pedrycz 1983; Buckley 1985; Chang 1996). The triangular fuzzy number may be described as $\tilde{A}\left(a_{1}, a_{2}, a_{3}\right)$, which denotes that $\tilde{A}$ is a fuzzy set with membership $a_{1}, a_{2}$ and $a_{3}$, as illustrated in Figure 1 . Thus, $\mu_{\widetilde{A}}(x)$ refers to the degree of membership of element x in fuzzy set $A$, which consists of a set of numbers $\left(a_{1}, a_{2}, a_{3}\right)$ in the interval $[0,1]$ (Tseng, Ding \& Chang 2017). It is described by Ross (1995) as: 
$\mu \widetilde{\mathrm{A}}(x)\left\{\begin{array}{l}\mathrm{O}, x<a_{1} \\ \frac{x-a_{1}}{a_{2}-a_{1}}, a_{1} \leq x \leq a_{2} \\ \frac{a_{3}-x}{a_{3}-a_{2}}, a_{2} \leq x \leq a_{3} \\ 0, x>a_{3}\end{array}\right\}$

Figure 1 shows a triangular fuzzy number represented by the triplet $a_{1}, a_{2}$ and $a_{3}$.

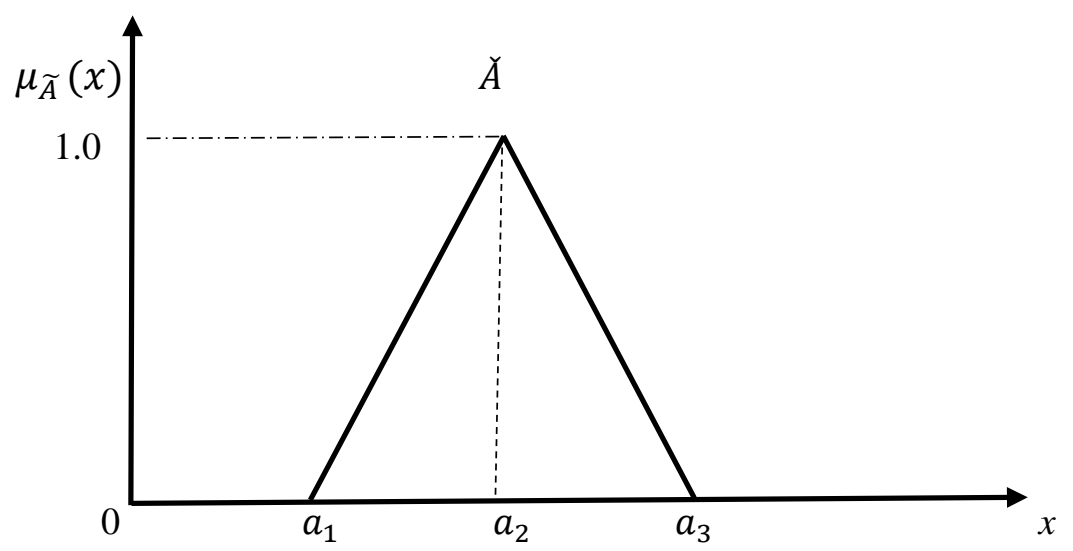

Figure: 1 Membership as the triangle fuzzy number

Figure 2 shows a triangular fuzzy number as $\tilde{A}=(\delta, b, \alpha)$ with two sets of fuzzy numbers $\tilde{A}_{1}=\left(\delta_{1}, b_{1}, \alpha_{1}\right)$ and $\tilde{A}_{2}=\left(\delta_{2}, b_{2}, \alpha_{2}\right)$, which can be computed as follows (Chang 1996; Chou 2008; Kwong \& Bai 2003; Tseng 2017):

- $\tilde{\mathrm{A}}_{1}+\tilde{\mathrm{A}}_{2}=\left(\delta_{1}+\delta_{2}, b_{1}-b_{2}, \alpha_{1}-\alpha_{2}\right)$

- $\tilde{\mathrm{A}}_{1}-\tilde{\mathrm{A}}_{2}=\tilde{\mathrm{A}}_{1}+\left(-\tilde{\mathrm{A}}_{2}\right)=\left(\delta_{1}-\delta_{2}, b_{1}-b_{2}, \alpha_{1}-\alpha_{2}\right)$

- $\widetilde{\mathrm{A}}_{1} \times \widetilde{\mathrm{A}}_{2}=\left(\delta_{1} \delta_{2}, b_{1} b_{2}, \alpha_{1} \alpha_{2}\right)$

- $\mathrm{K} \times \tilde{\mathrm{A}}_{1}=\left(\mathrm{K} \delta_{1}, \mathrm{~K} b_{1}, \mathrm{~K} \alpha_{1}\right)$

- $\mathrm{A}^{-1}=\left(\frac{1}{\alpha}, \frac{1}{b}, \frac{1}{\delta}\right)$

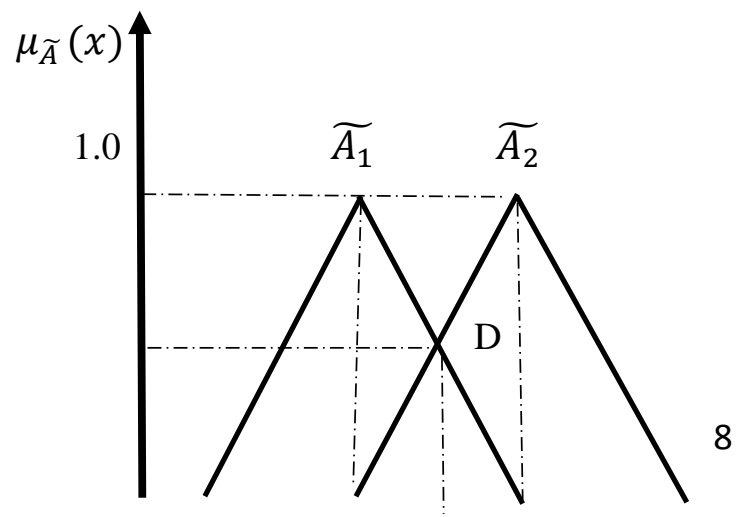




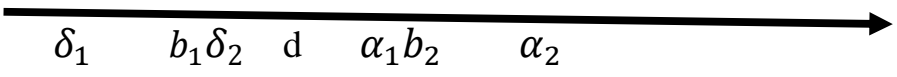

Figure: 2 Membership of two sets of triangular fuzzy numbers

The concept of fuzzy membership function was adopted in this study to evaluate the significance of human factors that may have an impact on human-centred performance using the mean values obtained as shown in Table 2 . The mean values are classified and described by linguistic terms as absolutely important, very strongly important, strongly important, and weakly important and equally important, respectively. Column 1 and 2 in Table 3 shows a classification of mean value and linguistic term in response to the triangular fuzzy scale and the triangular fuzzy reciprocal scale in column 3, and 4 respectively. Figure 3 shows the degree of membership in triangular fuzzy numbers corresponding to the linguistic terms.

Table 3 Mean values and linguistic terms vs trangualar fuzzy scale/triangular fuzzy reciprocal scale

\begin{tabular}{llll}
\hline Mean & Linguistic terms & $\begin{array}{l}\text { Triangular } \\
\text { fuzzy scale }\end{array}$ & $\begin{array}{l}\text { Triangular fuzzy } \\
\text { reciprocal scale }\end{array}$ \\
\cline { 2 - 4 } & Equal & $(1,1,1)$ & $(1,1,1)$ \\
\cline { 2 - 4 } & Equally important (EI) & $(1 / 2,1,3 / 2)$ & $(1 / 2,1,3 / 2)$ \\
\hline $3.5-3.7$ & Weakly important (WMI) & $(1,3 / 2,2)$ & $(1 / 2,2 / 3,1)$ \\
\hline $3.8-4.0$ & Strongly important (SMI) & $(3 / 2,2,5 / 2)$ & $(2 / 5,1 / 2,2 / 3)$ \\
\hline $4.1-4.3$ & Very strongly important (VSMI) & $(2,5 / 2,3)$ & $(1 / 3,2 / 5,1 / 2)$ \\
\hline $4.4-5.0$ & Absolutely important (AMI) & $(5 / 2,3,7 / 2)$ & $(2 / 7,1 / 3,2 / 5)$ \\
\hline
\end{tabular}

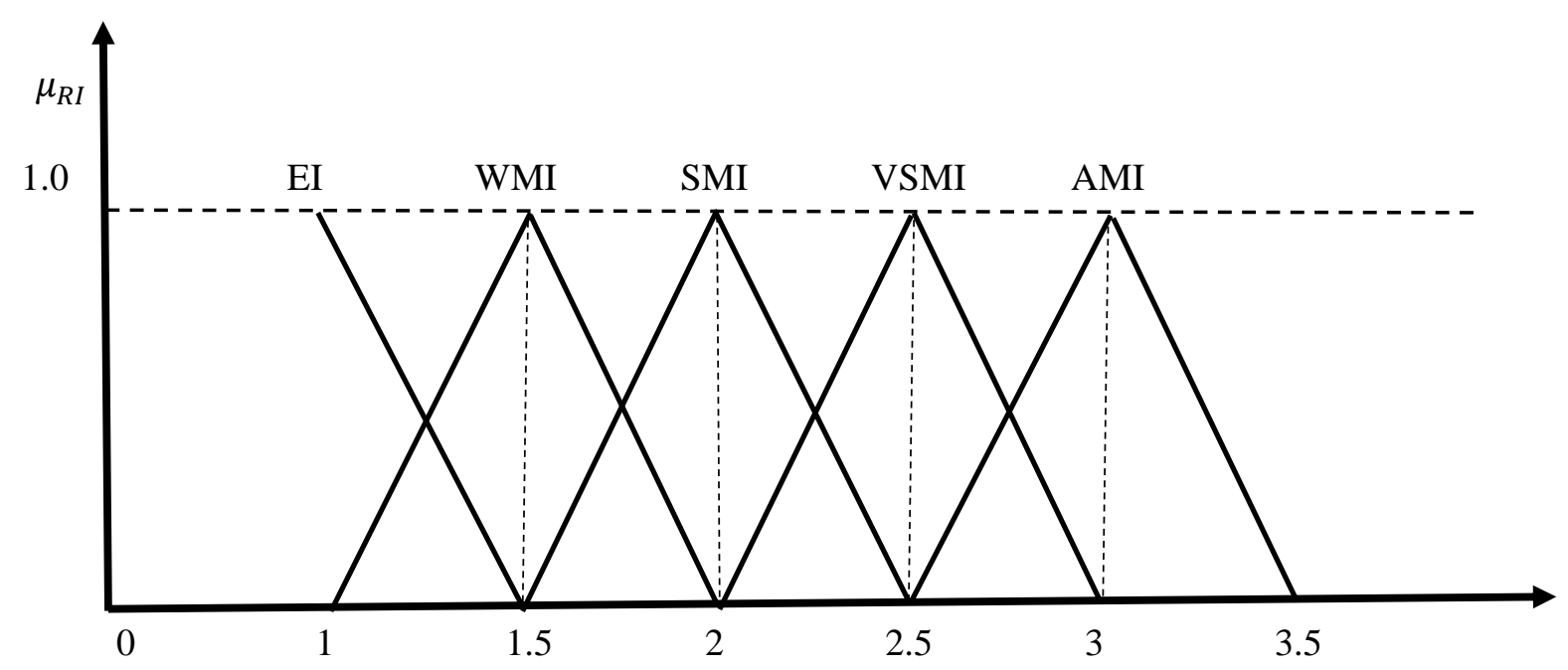

Figure: 3 Degree of membership of triangular fuzzy numbers

Table 4 shows the linguistic scale corresponding to the fuzzy numbers for rating the linguistic terms. 
Table 4 linguistic scale corresponding to the fuzzy numbers for rating the linguistic terms

\begin{tabular}{ll}
\hline Linguistic scale & fuzzy numbers \\
\hline Very high (VH) & $(0.25,0.5,0.75)$ \\
\hline High (H) & $(0,0.25,0.5)$ \\
\hline Low $(\mathrm{L})$ & $(0,0.25,0.25)$ \\
\hline
\end{tabular}

Table 5 shows a fuzzy decision matrix on which elements are transformed into triangular fuzzy numbers.

Table 5 Decision matrix using the triangular fuzzy numbers

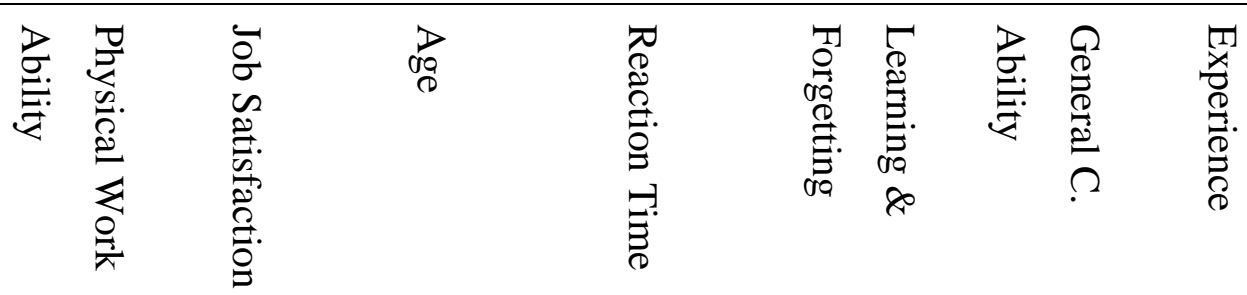

\begin{tabular}{|c|c|c|c|c|c|c|c|}
\hline Physical W. C. & $(1,1,1)$ & $(3 / 2,2,5 / 2)$ & $(1 / 3,2 / 5,1 / 2)$ & $(1 / 2,1,3 / 2)$ & $(1 / 2,1,3 / 2)$ & $(1 / 2,1,3 / 2)$ & $(1 / 3,2 / 5,1 / 2)$ \\
\hline Job S. & $(2 / 5,1 / 2,2 / 3)$ & $(1,1,1)$ & $(2 / 5,1 / 2,2 / 3)$ & $(1 / 2,1,3 / 2)$ & $(1 / 2,1,3 / 2)$ & $(1 / 2,1,3 / 2)$ & $(1 / 2,1,3 / 2)$ \\
\hline Age & $(2,5 / 2,3)$ & $(3 / 2,2,5 / 2)$ & $(1,1,1)$ & $(3 / 2,2,5 / 2)$ & $(1,3 / 2,2)$ & $(3 / 2,2,5 / 2))$ & $(2 / 7,1 / 3,2 / 5)$ \\
\hline Reaction Time & $(2 / 3,1,2)$ & $(2 / 3,1,2)$ & $(2 / 5,1 / 2,2 / 3)$ & $(1,1,1)$ & $(1 / 2,1,3 / 2)$ & $(2 / 5,1 / 2,2 / 3)$ & $(1 / 3,2 / 5,1 / 2)$ \\
\hline Learning \& F & $(2 / 3,1,2)$ & $(2 / 3,1,2)$ & $(1 / 2,2 / 3,1)$ & $(1 / 2,1,3 / 2)$ & $(1,1,1)$ & $(1 / 3,2 / 5,1 / 2)$ & $(2 / 5,1 / 2,2 / 3)$ \\
\hline General C. A. & $(2 / 3,1,2)$ & $(2 / 3,1,2)$ & $(2 / 5,1 / 2,2 / 3)$ & $(3 / 2,2,5 / 2)$ & $(2,5 / 2,3)$ & $(1,1,1)$ & $(2 / 5,1 / 2,2 / 3)$ \\
\hline
\end{tabular}

\subsection{Fuzzy Extent analysis}

In this study, an extent analysis method was used, which is an extension of fuzzy analytical hierarchy process (FAHP). The fuzzy extent analysis approach was used as a multi-criteria decision tool (Isik \& Aladag 2017; Yuksel \& Dagdeviren 2008; Divesh et al. 2017; Kahraman et al. 2006; Aggarwal \& Singh 2013; Adebanjo et al. 2017). Extent analysis 
method is applied in decision making with objective set $K$ towards a goal set $G=\left(g_{1}, g_{2}, g_{3} \ldots \ldots \ldots . g_{m}\right)$

Let $\mathrm{K}_{g i}^{1} \mathrm{~K}_{g i}^{2} \ldots . . \mathrm{K}_{g i}^{n}$ be an objects set towards a goal set $G=\left(g_{1}, g_{2}, g_{3} \ldots \ldots \ldots . . g_{n}\right)$, i.e., each individual object is performed towards each goal g, respectively. The value for each alternative decision can be obtained using the extent analysis described below (Chang 1996; Kahraman 2006; Tolga 2005):

$$
\tilde{\mathbf{M}}=\sum_{j=1}^{m} \mathbf{K}_{g i}^{j}\left(\sum_{i=1}^{n} \sum_{j=1}^{m} \mathbf{K}_{g i}{ }^{j}\right)^{-1}
$$

where $K_{g i}^{j}(j=1,2,3 \ldots \ldots n)$ are the triangular fuzzy numbers. To obtain $\sum_{i=1}^{n} \mathrm{~K}_{g i}^{j}$, we perform the fuzzy addition of m extent analysis for a particular matrix, which is given:

$\sum_{i=1}^{n} \mathrm{~K}_{g i}^{j}=\left(\sum_{i=1}^{n} \boldsymbol{l}_{j}, \sum_{i=1}^{n} \boldsymbol{m}_{j}, \sum_{i=1}^{n} \boldsymbol{u}_{j}\right)$

And, to obtain $\left(\sum_{i=1}^{n} \sum_{j=1}^{m} \mathrm{~K}_{g i}{ }^{j}\right)^{-1}$, operation for addition of fuzzy numbers is performed on

$\mathrm{K}_{g i}^{j}(j=1,2,3 \ldots n)$ such that

$$
\left.\left(\sum_{i=1}^{n} \sum_{j=1}^{m} \mathrm{~K}_{g i}^{j}\right)^{-1}=\left(\frac{1}{\sum_{i=1}^{n} u_{1}}, \frac{1}{\sum_{i=1}^{n} m_{1}}, \frac{1}{\sum_{i=1}^{n} l_{1}}\right) \text { Where } \forall l_{1}, m_{1}, u_{1}\right\rangle 0
$$

Table 6 shows the calculated synthetic extent values for the decision alternatives using equations 7 to 9 , where $l, m, u$ denotes the lower, medium and upper bounds of the degree of membership.

Table 6 Synthetic extent values computed for each of decision alternatives

\begin{tabular}{lccc}
\hline & $l$ & $m$ & $u$ \\
\hline$S_{\text {physical work capacity }\left(S_{p w c}\right)}$ & 0.07 & 0.11 & 0.21 \\
\hline$S_{\text {job satisfaction }}\left(S_{j s}\right)$ & 0.06 & 0.10 & 0.19 \\
\hline$S_{\text {age }}\left(S_{a g}\right)$ & 0.13 & 0.19 & 0.32 \\
\hline$S_{\text {reaction time }}\left(S_{r t}\right)$ & 0.06 & 0.09 & 0.19 \\
\hline$S_{\text {learning and forgetting }}\left(S_{l f}\right)$ & 0.06 & 0.09 & 0.21 \\
\hline$S_{\text {general cognitive ability }}\left(S_{\text {gca }}\right)$ & 0.10 & 0.14 & 0.40 \\
\hline$S_{\text {experience }}\left(S_{\text {exp }}\right)$ & 0.17 & 0.24 & 0.37 \\
\hline
\end{tabular}


The estimation for sets of weight values under each criterion was obtained by comparing fuzzy numbers $K_{1}$ and $K_{2}$. And whether the degree of possibility of $K_{1}$ is greater or equal to $K_{2}$ is determined by the intersection of the two fuzzy numbers $\tilde{\mathrm{K}}_{2}\left(l_{2}, m_{2}, u_{2}\right)$ and $\tilde{\mathrm{K}}_{1}\left(l_{1}, m_{1}, u_{1}\right) \cdot v\left(\tilde{\mathrm{K}}_{1} \geq \tilde{\mathrm{K}}_{2}\right)=1$, since $\tilde{\mathrm{K}}_{1}$ and $\tilde{\mathrm{K}}_{2}$ are convex set fuzzy numbers (Zhu, 1999), thus, it can be expressed as:

$$
\vee\left(\tilde{\mathrm{K}}_{1} \geq \tilde{\mathrm{K}}_{2}\right)=\operatorname{hgt}\left(\tilde{\mathrm{K}}_{1} \geq \tilde{\mathrm{K}}_{2}\right)=\vee\left(\tilde{\mathrm{K}}_{1} \cap \mathrm{K}_{2}\right)=\left(\frac{l_{1}-u_{2}}{\left(m_{2}-u_{2}\right)-\left(m_{1}-l_{1}\right)}\right)=\mu \mathrm{K}(d)
$$

And this can be represented as:

$$
\begin{aligned}
& \vee\left(\mathrm{K}_{1} \geq \mathrm{K}_{2}\right)=\mu_{A}(d)=\left\{\begin{array}{l}
1 i f f m_{2} \geq m_{1} \\
0 i f f l_{1} \geq u_{2} \\
\frac{l_{1}-u_{2}}{\left(m_{2}-u_{2}\right)-\left(m_{1}-l_{1}\right)}
\end{array}\right. \text { otherwise } \\
& \vee\left(\mathrm{K}_{1} \geq \mathrm{K}_{2}\right)=1 \text { iff } \tilde{\mathrm{K}}_{1}=\tilde{\mathrm{K}}_{2}
\end{aligned}
$$

Where, if and only if $d$ is the ordinate of the highest intersection D between $\mu \tilde{K}_{1}$ and $\mu \tilde{\mathrm{K}}_{2}$ (see Figure 4) (Zhu 1999).

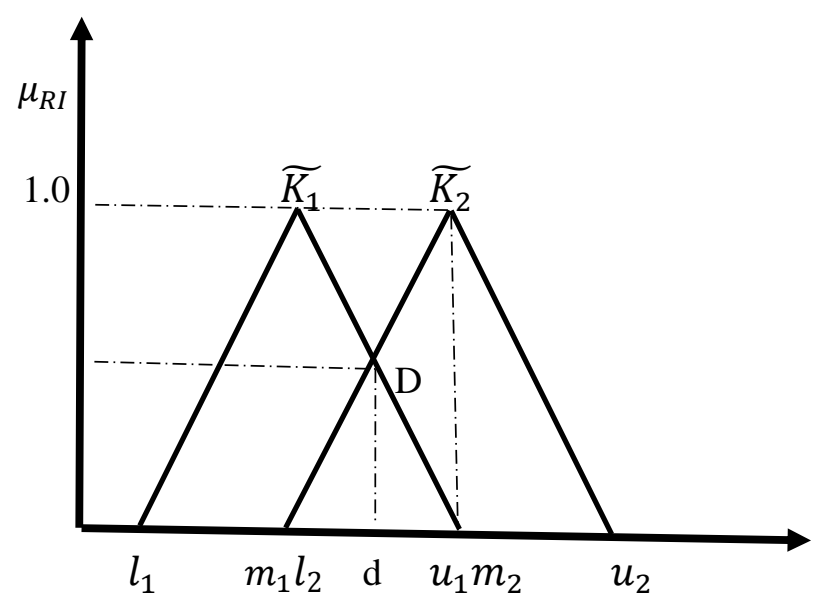

Figure: 4 The degree of intersection of two fuzzy sets

Using equations 10 and 11, Table 7 shows the weight vector for each decision alternative.

Table 7 Weight vector for each decision alternative 


\begin{tabular}{|c|c|c|c|c|c|c|c|}
\hline $\mathrm{V}\left(S_{p w c} \geq S_{j s}\right)$ & 1 & $\mathrm{~V}\left(S_{j s} \geq S_{p w c}\right)$ & 1 & $\mathrm{~V}\left(S_{a g} \geq S_{p w c}\right)$ & 1 & $\mathrm{~V}\left(S_{r t} \geq S_{p w c}\right)$ & 0.91 \\
\hline $\mathrm{V}\left(S_{p w c} \geq S_{a g}\right)$ & 0.53 & $\mathrm{~V}\left(S_{j s} \geq S_{a g}\right)$ & 0.56 & $\mathrm{~V}\left(S_{a g} \geq S_{j s}\right)$ & 1 & $\mathrm{~V}\left(S_{r t} \geq S_{j s}\right)$ & 0.92 \\
\hline $\mathrm{V}\left(S_{p w c} \geq S_{r t}\right)$ & 1 & $\mathrm{~V}\left(S_{j s} \geq S_{r t}\right)$ & 1 & $\mathrm{~V}\left(S_{a g} \geq S_{r t}\right)$ & 1 & $\mathrm{~V}\left(S_{r t} \geq S_{a g}\right)$ & 0.42 \\
\hline $\mathrm{V}\left(S_{p w c} \geq S_{l f}\right)$ & 1 & $\mathrm{~V}\left(S_{j s} \geq S_{l f}\right)$ & 1 & $\mathrm{~V}\left(S_{a g} \geq S_{l f}\right)$ & 1 & $\mathrm{~V}\left(S_{r t} \geq S_{l f}\right)$ & 1 \\
\hline $\mathrm{V}\left(S_{p w c} \geq S_{g c a}\right)$ & 1 & $\mathrm{~V}\left(S_{j s} \geq S_{g c a}\right)$ & 1 & $\mathrm{~V}\left(S_{a g} \geq S_{g c a}\right)$ & 1 & $\mathrm{~V}\left(S_{r t} \geq S_{g c a}\right)$ & 0.90 \\
\hline $\mathrm{V}\left(S_{p w c} \geq S_{e x p}\right)$ & 0.35 & $\mathrm{~V}\left(S_{j s} \geq S_{\text {exp }}\right)$ & 0.38 & $\mathrm{~V}\left(S_{a g} \geq S_{e x p}\right)$ & 0.81 & $\mathrm{~V}\left(S_{r t} \geq S_{\text {exp }}\right)$ & 0.25 \\
\hline $\mathrm{V}\left(S_{p w c} \geq S_{e f}\right)$ & 0.80 & $\mathrm{~V}\left(S_{j s} \geq S_{e f}\right)$ & 0.81 & $\mathrm{~V}\left(S_{a g} \geq S_{e f}\right)$ & 1 & $\mathrm{~V}\left(S_{r t} \geq S_{e f}\right)$ & 0.71 \\
\hline $\mathrm{V}\left(S_{l f} \geq S_{p w c}\right)$ & 0.80 & $\mathrm{~V}\left(S_{g c a} \geq S_{p w c}\right)$ & 1 & $\mathrm{~V}\left(S_{\text {exp }} \geq S_{p w c}\right.$ & 1 & $\mathrm{~V}\left(S_{e f} \geq S_{p w c}\right)$ & 1 \\
\hline $\mathrm{V}\left(S_{l f} \geq S_{j s}\right)$ & 0.92 & $\mathrm{~V}\left(S_{g c a} \geq S_{j s}\right)$ & 1 & $\mathrm{~V}\left(S_{\text {exp }} \geq S_{j s}\right)$ & 1 & $\mathrm{~V}\left(S_{e f} \geq S_{j s}\right)$ & 1 \\
\hline $\mathrm{V}\left(S_{l f} \geq S_{a g}\right)$ & 0.46 & $\mathrm{~V}\left(S_{\exp } \geq S_{a g}\right)$ & 0.66 & $\mathrm{~V}\left(S_{e x p} \geq S_{a g}\right)$ & 1 & $\mathrm{~V}\left(S_{e f} \geq S_{a g}\right)$ & 0.78 \\
\hline $\mathrm{V}\left(S_{l f} \geq S_{r t}\right)$ & 1 & $\mathrm{~V}\left(S_{g c a} \geq S_{r t}\right)$ & 1 & $\mathrm{~V}\left(S_{\text {exp }} \geq S_{r t}\right)$ & 1 & $\mathrm{~V}\left(S_{e f} \geq S_{r t}\right)$ & 1 \\
\hline $\mathrm{V}\left(S_{l f} \geq S_{g c a}\right)$ & 0.90 & $\mathrm{~V}\left(S_{g c a} \geq S_{l f}\right)$ & 1 & $\mathrm{~V}\left(S_{\text {exp }} \geq S_{l f}\right)$ & 1 & $\mathrm{~V}\left(S_{e f} \geq S_{l f}\right)$ & 1 \\
\hline $\mathrm{V}\left(S_{l f} \geq S_{\text {exp }}\right)$ & 0.29 & $\mathrm{~V}\left(S_{g c a} \geq S_{e x p}\right)$ & 0.52 & $\mathrm{~V}\left(S_{\text {exp }} \geq S_{g c a}\right)$ & 1 & $\mathrm{~V}\left(S_{e f} \geq S_{g c a}\right)$ & 1 \\
\hline $\mathrm{V}\left(S_{l f} \geq S_{e f}\right)$ & 0.73 & $\mathrm{~V}\left(S_{g c a} \geq S_{e f}\right)$ & 0.85 & $\mathrm{~V}\left(S_{e x p} \geq S_{e f}\right)$ & 1 & $\mathrm{~V}\left(S_{e f} \geq S_{e x p}\right)$ & 0.60 \\
\hline
\end{tabular}

The degree of possibility for a convex set fuzzy number to be greater than another convex set fuzzy numbers $M_{i}(i=1,2,3,4,5,6, \ldots \ldots k)$ is given as (Kahraman 2006; Tolga $2005 \&$ Zhu 1999):

$\vee\left(M \geq M_{1}, M_{2}, M_{3} \ldots . . M_{k}\right)=\vee\left[\left(M \geq M_{1}\right),\left(M \geq M_{2}\right) \ldots . .\left(M \geq M_{n}\right)\right]$

$=\min \vee\left(\mathrm{M} \geq \mathrm{M}_{i}\right), i=1,2,3 \ldots \ldots \ldots \ldots . . . n$

Assuming that

$\mathrm{A}_{i} \min \vee\left(S_{1} \geq S_{k}\right)$ for $\mathrm{k}=1 \ldots 3 \ldots 5 \ldots \mathrm{n} \quad \mathrm{k} \neq i$.

Thus, the weight vector $\mathrm{W}$ is:

$W^{\prime}=\left[d^{\prime}\left(\mathrm{A}_{1}\right), d^{\prime}\left(\mathrm{A}_{2}\right), d^{\prime}\left(\mathrm{A}_{3}\right), d^{\prime}\left(\mathrm{A}_{4}\right) \ldots \ldots . d^{\prime}\left(\mathrm{A}_{n}\right)\right]$

Where, $A_{i} \in n$

The normalized weight vectors W (non-fuzzy number) can be gained below: 
$\mathrm{W}=\left(d^{\prime}\left(A_{1}\right), d^{\prime}\left(A_{2}\right), d^{\prime}\left(A_{3}\right), d^{\prime}\left(A_{4}\right), \ldots \ldots d^{\prime}\left(A_{n}\right)\right)$

Where, $d^{\prime}\left(A_{1}\right) \ldots . d^{\prime}\left(A_{n}\right)$ are decision alternatives

Table 8 shows the minimum weight vectors for each decision alternative, which were obtained from equation 12 to 13 (Chang 1996).

Table 8 The minimum decision vector for each decision alternative

$\mathrm{d}^{\prime}\left(A_{1}\right) \quad \mathrm{V}\left(S_{p w c} \geq S_{j s .} S_{a g .} S_{r t .} S_{l f .} S_{g c a .} S_{\text {exp. }}\right)=\min (1,0.47,1,1,0.78,0.21)=0.21$

\begin{tabular}{|c|c|}
\hline $\mathrm{d}^{\prime}\left(A_{2}\right)$ & $\mathrm{V}\left(S_{j s} \geq S_{p w c .} S_{a g .} S_{r t .} S_{l f .} S_{g c a .} S_{\text {exp. }}\right)=\min (0.89,0.37,1,1,0.67,0.11)=0.11$ \\
\hline $\mathrm{d}^{\prime}\left(A_{3}\right)$ & $\left(\vee S_{a g} \geq S_{p w c .} S_{j s .} S_{r t .} S_{l f .} S_{g c a .} S_{\text {exp. }}\right)=\min (1,1,1,1,1,0.76)=0.76$ \\
\hline $\mathrm{d}^{\prime}\left(A_{4}\right)$ & $\mathrm{V}\left(S_{r t} \geq S_{p w c .} S_{j s .} S_{a g .} S_{l f .} S_{g c a .} S_{\text {exp. }}\right)=\min (0.93,1,0.35,1,0.62,0.11)=0.11$ \\
\hline $\mathrm{d}^{\prime}\left(A_{5}\right)$ & $\mathrm{V}\left(S_{l f} \geq S_{p w c .} S_{j s .} S_{a g .} S_{r t .} S_{l f .} S_{\text {exp. }}\right)=\min (1,1,0.42,1,0.69,0.2)=0.20$ \\
\hline $\mathrm{d}^{\prime}\left(A_{6}\right)$ & $\mathrm{V}\left(S_{g c a} \geq S_{p w c .} S_{j s .} S_{a g .} S_{r t .} S_{l f}, S_{\text {exp. }}\right)=\min (1,1,0.73,1,1,0.51)=0.51$ \\
\hline $\mathrm{d}^{\prime}\left(A_{7}\right)$ & $\mathrm{V}\left(S_{\text {exp }} \geq S_{p w c .} S_{j s .} S_{a g .} S_{r t .} S_{l f .} S_{g c a .}\right)=\min (1,1,1,1,1,1)=1$ \\
\hline The weight decision alternative & $\mathrm{W}=(0.21,0.11,0.76,0.11,0.20,0.51,1)$ \\
\hline $\begin{array}{l}\text { based on their influence and the } \\
\text { normalization. }\end{array}$ & $\mathrm{W}=(0.07,0.04,0.26,0.04,0.07,0.18,0.34)$ \\
\hline
\end{tabular}

Figure 5 illustrates the importance of each decision alternative using the extent analysis procedure. It ranks experience as the most critical human factor that affects human-centred performance as it accounts for $34 \%$ of the effect, followed by age (26\%), general cognitive abilities (18\%), physical work capacity (7\%), learning and forgetting (7\%), reaction time (4\%) and job satisfaction (4\%), which have the least effect on human performance. 


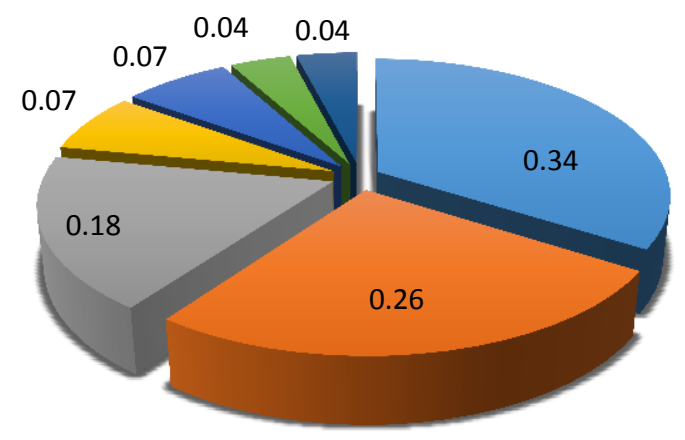

\begin{tabular}{|c|c|c|}
\hline Experience & Age & General cognitive abilities $\square$ Physical work capacity \\
\hline Learning and forgetting & Reaction time & Job satisfaction \\
\hline
\end{tabular}

Figure: 5 Synthesized priorities of human factors affecting human-centred performance The significance of each of human factors was converted into a global weight as shown in Table 9.

Table 9 Rating of human factors significance on human-centred assembly performance

\begin{tabular}{lll}
\hline Human Factors & Global weight & Level of influence \\
\hline Experience & 1 & Very high \\
Age & 0.76 & Very high \\
General cognitive abilities & 0.51 & High \\
Physical work capacity & 0.21 & Low \\
Learning and forgetting & 0.20 & Low \\
Reaction time & 0.11 & Low \\
Job satisfaction & 0.11 & Low \\
\hline
\end{tabular}

According to the study, one of critical human factors that significantly affect human-centred assembly performance is the effect of ageing. Ageing workers are defined by the International Labour Organisation (ILO) as workers who are liable to encounter difficulties in employment and occupation because of advancement in age (Asogwa et al. 1993). Gerontologists classified the ageing population into three groups: group at ages between 60 to 74 years old, group at ages between 75 to 85 years old, and group at ages of 80 years old and beyond (Mendonca 2017). Whilist World Health Organisation (WHO) defines an ageing person older than 45 years old (Asogwa et al. 1993; Peruzzini \& Pellicciari, 2017). Regardless of various interpretations, the percentage of older workers at ages between 55 to 
64 years have risen by $50 \%$ in the past two decades, and may continue to rise (Boenzi et al. 2015). Ageing can cause the persistant decline in the biological components due to the internal physiological deterioration (Kenny et al. 2015). There is evidence that individual performance may decline from a certain age due to the natural decline of physical and physiological functions, such as visual ability, musculoskeletal force, flexibility/motion capability, memory/concentration and thermoregulation (Skirbekk 2008; Peruzzini \& Pellicciari 2017; Robertson \& Tracy 1998).

In manufacturing, assembly by human workers involve operations of pinching, gripping, screwing, pulling, pushing, lifting, turning and so on. It requires repetitive wrist motions and hand postures, which may be associated with tendon disorders of hands and wrists leading to loss of productivity due to pain and fatigue (Palmer et al. 2006). Studies indicated that job performance of most individuals may increase until the age of 35 years old; after this age it may steadily decline. It can decline up to $25 \%$ at ages from 45 to 64 years old (Skirbekk 2008; Peruzzini \& Pellicciari 2017). Zwick \& Gobel (2009) investigated the effects of ageing on productivity and observed that productivity may increase until the age of 40 years old; with more significant decline after the age of 60 years old. However, Leyk et al. (2010) argued that losses in human performance are primarily related to a sedentary lifestyle rather than a chronological number of years. Generally, female workers are physically weaker than male workers and strength of female workers is approximately two thirds of male workers. Nevertheless, as worker's age increases, it is expected that their experience also increases. And this may offset the decline of human abilities (Warr 1994; Salthaouse \& Somberg 1982; Giniger 1983). In this study, however, it was assumed that human natural decline of physical, physiological and cognitive systems over increase of age is unavoidable, subjected to individual habitual adaptation (such as poor health, inactive lifestyle, smoking, poor diet, substance abuse and so on). And the ability of an organizations to accommodate and protect workforce particularly in the context of employment standards (Chaffin 2006; Skirbekk, 2008). Table 10 shows a summary of findings through a literature review relating to the decline of human functons over age.

Table 10 Literature studies in decline on of human functional abilities over age

\begin{tabular}{lllll}
\hline Ability & Functions & Performance variations & Authors \\
\hline Endurance & Aerobic & Peak at the age of 40 and decline by 1\% per & Robertson \& \\
& capacity & year after this age & Tracy 1998 & \\
\hline
\end{tabular}




\begin{tabular}{|c|c|c|c|}
\hline & & $\begin{array}{l}\text { Peak at the age of } 30 \text { and decline by } 0.5- \\
1.5 \% \text { per year }\end{array}$ & $\begin{array}{l}\text { Bellew 2005; } \\
\text { Savinainen } \\
\text { 2004a }\end{array}$ \\
\hline & & $\begin{array}{l}\text { Decline by } 1-1.5 \% \text { per year after the age } \\
\text { of } 40\end{array}$ & $\begin{array}{l}\text { Boenzi et al. } \\
2015\end{array}$ \\
\hline & & Decline by $1 \%$ per year after the age 30 & $\begin{array}{l}\text { Hawkins \& } \\
\text { Wiswel } 2003\end{array}$ \\
\hline & & Decrease by $1 \%$ per year after the age of 35 & Crawford 2010 \\
\hline \multirow[t]{5}{*}{ Psychomotor } & $\begin{array}{l}\text { Spatial } \\
\text { ability }\end{array}$ & $\begin{array}{l}\text { Peak at the age of } 30 \text { and decline at } 1 \% \\
\text { per year after this age }\end{array}$ & Ellis et al. 1999 \\
\hline & & $\begin{array}{l}\text { Peak at the age of } 40 \text { and decline between } \\
0.8 \text { and } 1.0 \% \text { per year after this age }\end{array}$ & Basu 2002 \\
\hline & & $\begin{array}{l}\text { Peak at the age of } 30 \text {, decline by } 0.5 \% \text { per } \\
\text { year up to the age of } 40 \text { and then decline by } \\
1 \% \text { every yeat up to the age of } 65\end{array}$ & $\begin{array}{l}\text { Aoyagi 1992; } \\
\text { Gall } 2004\end{array}$ \\
\hline & & $\begin{array}{l}\text { Peak at the age of } 45 \text { and decline by } 1- \\
1.5 \% \text { per year after this age }\end{array}$ & $\begin{array}{l}\text { Savinainen } \\
2004 b\end{array}$ \\
\hline & & $\begin{array}{l}\text { Peak at the age of } 40 \text { and decline by } 0.8- \\
1 \% \text { per year after this age }\end{array}$ & Fleg et al. 2005 \\
\hline $\begin{array}{l}\text { Akward } \\
\text { posture }\end{array}$ & Flexibility & $\begin{array}{l}\text { Peak at the age of } 35 \text { and decrease at about } \\
1.0 \% \text { per year from } 35 \text { to } 54\end{array}$ & Alaranta 1994 \\
\hline $\begin{array}{l}\text { Overall } \\
\text { performance }\end{array}$ & $\begin{array}{l}\text { Physiologic } \\
\text { al function }\end{array}$ & $\begin{array}{l}\text { Peak at the age of } 35-40 \text { and decline by } \\
1 \% \text { per year after these ages }\end{array}$ & $\begin{array}{l}\text { Asogwa 1993; } \\
\text { Savinainen } \\
\text { 2004; Ilmarinen } \\
2002\end{array}$ \\
\hline
\end{tabular}

\section{Numerical result and analysis}

Based on studies shown in Table 10, Table 11 shows the remaining functional capacity (\%) of his/her peak at age 38 due to human functional decline over varying ages using the regression analysis equation 16. It shows that human performance starts to deteriorate at 38 years old as a base line. It also shows loss rate, which refers to the rate of decline after the age of 38 years old. Figure 6 shows the trend in decline of human functional capacity at varying ages after 38 years old. 
$L_{r}=0.57+0.012 k_{1}$

(16)

Where

$L_{r}=$ Loss rate in percentage

$\mathrm{k}=$ Age in years

$F_{r m}=k_{2}-L_{r}\left(k_{1}-38\right)$

(17)

Where:

$F_{r m}$ : Remaining capacity in percentage of the peak at 38 years old

$k_{2}$ : Peak capacity (100\%) at 38 years old

$L_{r}:$ Loss rate in percentage

$k_{1}$ : Existing age in years

Table 11 Human functional decline in percentage over age

Age Loss rate Remaining functional capacity (\%) of Human kinematic decline rate $\left(L_{r}\right) \quad$ his/her peak at age $38\left(F_{r m}\right)$ (\%) of his/her full capacity

\begin{tabular}{llll}
\hline 38 & 0 & 100 & 0 \\
\hline 40 & 1.05 & 97.9 & 2.01 \\
\hline 45 & 1.11 & 92.23 & 7.77 \\
\hline 50 & 1.17 & 85.96 & 14.04 \\
\hline 55 & 1.23 & 79.09 & 20.91 \\
\hline 60 & 1.29 & 71.62 & 28.38 \\
\hline 65 & 1.35 & 63.55 & 36.45 \\
\hline 70 & 1.41 & 54.88 & 45.12 \\
\hline 75 & 1.47 & 45.61 & 54.39 \\
\hline 80 & 1.53 & 35.74 & 64.26 \\
\hline
\end{tabular}

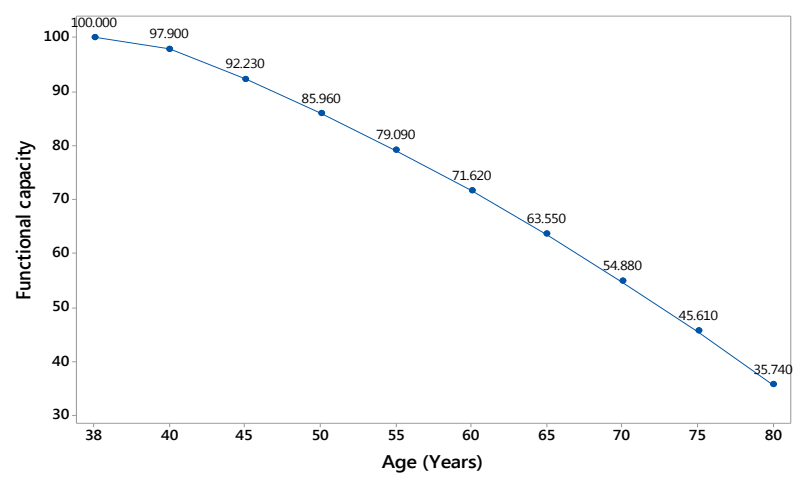

Figure: 6 Decline in human functional capacity after 38 years old 


\subsection{Learning effects}

One of learning effects refers to a trend in reduction of production time as quantity of products increases (Jaber \& Bonney 1997). Human learning pattern during production cycles can be represented by a log linear model (equation 18), which is widely used as a learning curve for predicting individuals' performance through a learning process (Jaber 2001):

$T_{n}=T_{t} \cdot Q^{c}$

Where

$T_{n}$ : Average time to produce the $n^{\text {th }}$ unit

$T_{t}$ : Assembly time to produce the first unit

Q: Cumulative number of units produced

C: Learning index which determines the speed of learning occurring each time as a cumulative output increases, it is computed as $\frac{\log (R)}{\log (2)}$ where the learning rate $\mathrm{R}$ is measured in percentage $(0<\mathrm{R}<1)$ (Anzanello \& Fogliatto, 2011). Note that the average time towards the steady state decreases with the increase in numbers of units produced. Thus, the average time towards a steady stage can be given as:

$\mathrm{T}_{\mathrm{A}}=\mathrm{T}_{t} \cdot \mathrm{B}^{R}$

or

$\mathrm{T}_{t}=\frac{\mathrm{T}_{\mathrm{A}}}{\mathrm{B}^{R}}$

Where

$T_{A}$ : The average-time towards a steady stage

B: Batch size

By substituting equation 20 into equation 18, it yields:

$\mathrm{T}_{n}=\frac{\mathrm{T}_{\mathrm{A}}}{\mathrm{B}^{R}} \cdot Q^{R}$

Hence 
$\mathrm{T}_{n}=\mathrm{T}_{\mathrm{A}}\left(\frac{Q}{\mathrm{~B}}\right)^{R}$

Figure 7 shows the trend of average assembly time corresponding to accumulative number of units produced by workers at the age of 38 years old during a learning process of repetitive operations of assembling a unit based on the result obtained using equation 22. It can be seen that the average assembly time tends to be stabilised after performing over 480 units.

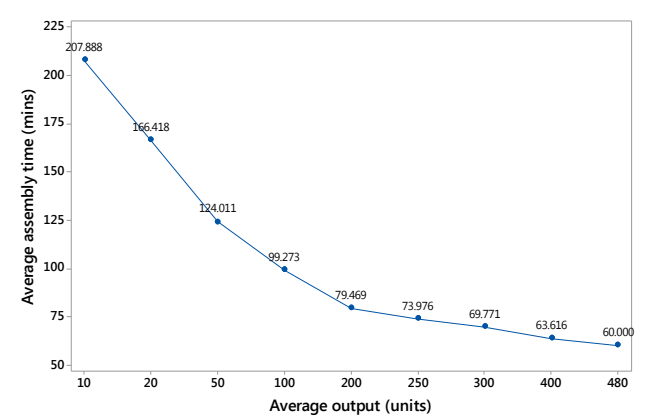

Figure: 7 Average assembly time (mins) vs accumulated output of workers at 38 years old

The loss in average assembly time per worker due to ageing is given below:
$\Delta_{L t}=$
$T_{n}$
$\times$
$F_{d l}$

(23)

Where

$F_{d l}$ : Kinematic decline rate (\%) of human full capacity (as shown in Table 11 )

$$
\Delta_{L T}=\mathrm{T}_{\mathrm{A}}\left(\frac{Q}{\mathrm{~B}}\right)^{R} \times F_{d l}
$$

Hence, average total assembly time per worker associated to ageing is computed below:

$\mathrm{T}_{\mathrm{A} t}=\mathrm{T}_{\mathrm{A}}\left(\frac{Q}{\mathrm{~B}}\right)^{R}+\mathrm{T}_{\mathrm{A}}\left(\frac{Q}{\mathrm{~B}}\right)^{R} \times F_{d l}$

Where

$\Delta_{L t}:$ Average assembly time loss due to ageing

$T_{A t}$ : Average total assembly time per worker due to ageing

\subsection{Analysis in a range of ageing groups}


Assuming an assembly line in which a worker has to assemble 480 units of a product and he/she is expected to be proficient at the job after 60 minutes. Figure 8 shows the average assembly time for each group of assembly workers over the accumulative number of assembled units at varying ages from 38 to 80 years old. Overall, it can be seen in Figure 9a that average assembly time drops over the increasing number of output (in units) for all the ageing groups. It can also be seen that average assembly time for the ageing group of 38 years old is 60 minutes, which is less than 64.66 minutes for the ageing group of 45 years old. Although there is an insignificant difference in average assembly time of the ageing groups between 38 and 40 years old. Figure $8 \mathrm{~b}$ and c show an average assembly time of the ageing groups from 50 to 80 years old. By comparing the average assembly time of the ageing group at 38 years old, it can be seen that the average assembly time increases approximate $1 \%$ per year from the ages of 38 to 70 years old and about $1.5 \%$ from the ages of 70 to 80 years old.

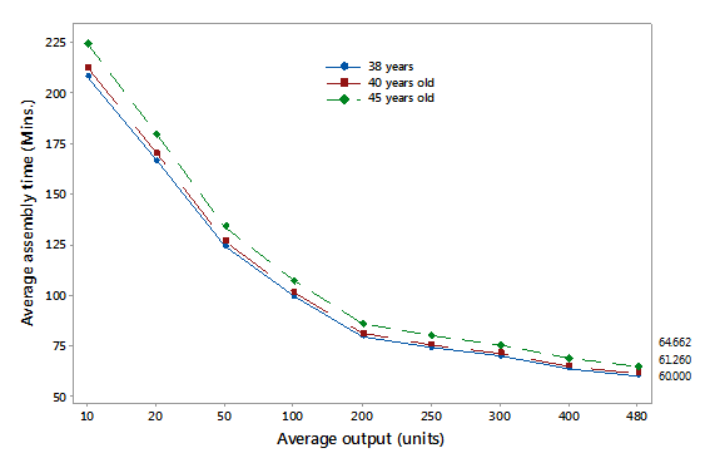

(a)

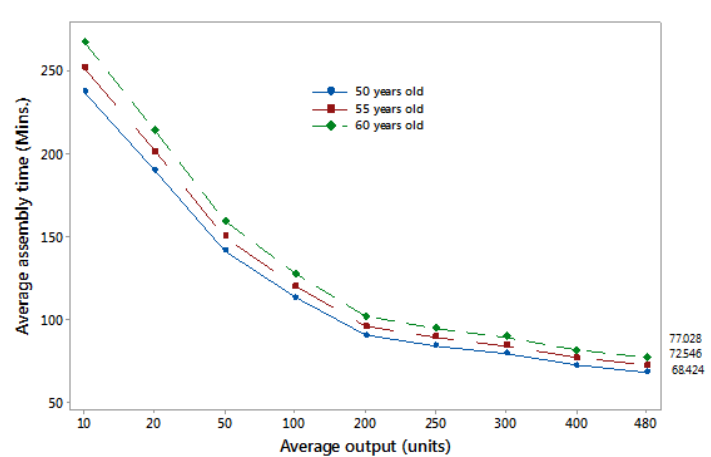

(b)

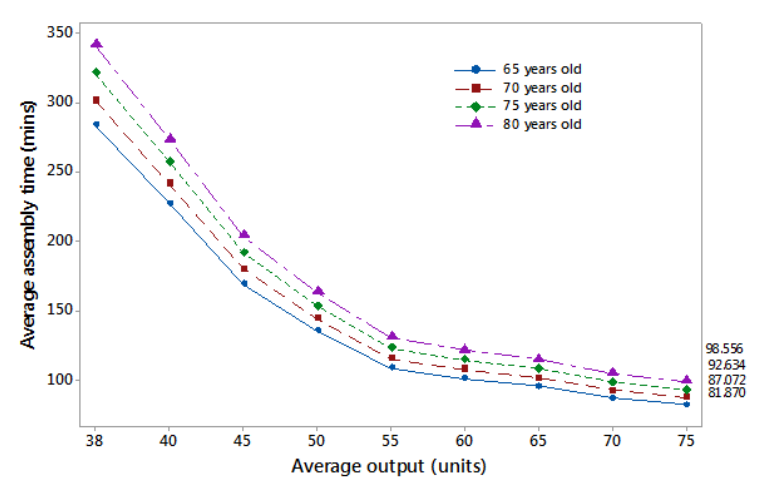

(c)

Figure: 8 Average assembly time of an ageing group of workers

\section{Summary and discussions}

Through the literature study, although there were some investigations into human factors relating to human performance, there were few studies by examining human factors and their 
interections on human performance of human-centred assembly systems. This paper presents a study by identifying the relevant human factors that may have impacts on human-centred assembly based on findings of the literature review and the industrial survey. This includes the proposed alternative methods used for quantifying the influential levels between one of key human factors and one of key performance indicators. The study shows that individual performances of human workers usually approach their full capacity at the age of 38 years old. After this age, it may decline over the increase of ages of workers. By contrast, the literature study also implies that work experience grows over the increase of age of workers leading to improvements of individual performance, which may also compensate the loss of assembly time of ageing workers. The study concludes age and experience are the most influential human factors that may affect human performance; this can be useful for line managers for decision makings in selection or evaluation of assembly workers for production. An on-going study is being carried out by incorporating the identified human factors into a DES (discrete even simulation) tool that is often applied as an aid for manufacturing systems design and evaluation. Current DES tools in the market do not have functionality allowing manufacturing systems designers to create a DES model that considers effects of human factors or human performance for production. This is because, for example, in a DES model, the workers are defined and treated as a simple resource the same as parts, machines, conveyors and so on. The application of DES simulation models is therefore restricted to predicting such variables as the required number of workers, their shift patterns and routes (Wang et al. 2012). These issues need also to be addressed more in future for DES packages in applications and development of manufacturing system related designs and operations.

\section{References}

Adam, J. J., Pass, F., Bueke, M. J., Wuyts, I. J., Spijkers, W., \& Wallmeyer, P. (1999). Gender differences in choice reaction time: evidence for differential strategies. Ergonomics, Vol. 42(2) 327-335.

Adebanjo, D., Laosirihongehong, T., \& Samaranayeke, P. (2017). Prioritizing lean supply chain management initiatives in health care services operations: Fuzzy AHP approach. Production Planning and Control: The Management Operations, Vol. 27(12), 953-966.

Aggarwal, R., \& Singh, S. (2013). AHP and extend fuzzy AHP approach for prioritizing of performance measurements attributes. International Journal of Mechanical, Aerospace, Industrial, Mechatronics and Manufacturing Engineering, Vol. 7(1), 6-11.

Alaranta, H., Hurri, H., Hallovaara, M., Soukka, A., \& Harju, R. (1994). Flexibility of the spine: normative values of geometric and tape measurement. Scandinavian Journal of Rehabilitation Medicine, Vol. 26(3), 147-154. 
Allen, I. E., \& Seaman, C. A. (2007). Likert Scales and data analysis. Quality Progress, 64-65.

Ando, S., Kida, N., \& Oda, S. (2004). Retention of practice effect on simple reaction time for peripheral and central visual fields. Ergonomics, Vol. 98, 897-900.

Anzanello, M. J., \& Fogliatto, F. S. (2011). Learning curve models and applications: literature review and research directions. International Journal of Industrial Ergonomics, Vol. 41, 573-583.

Aoyagi, Y., \& Shepherd, R. J. (1992). Aging and muscle function. Sports medicine, Vol. 14(6), 376-396.

Asogwa, S. E., Ibarra, A. G., Izmeron, N. F., Kumashiro, M., Ramachandra, C. R., Rantanen, J., \& Su, Z. (1993). Aging and Working Capacity. Geneva: World Health Organisation.

Avolio, B. J., \& Waldman, D. A. (1990). Age and work performance in Non-Managerial jobs: the effect of experience and occupational type . Academy of Management Journal, Vol. 33(2), 407 422.

Baines, T., Mason, S., Siebers, P. O., \& Ladbrook, J. (2004). Humans: The Missing Link in Manufacturing Simulation? Simulation Modelling Practice and Theory, vol.12, 515 - 526.

Bainess, T. S., \& Kay, J. M. (2002). Human performance modelling as aid in the process of manufacturing system design. International Journal of Production Research, Vol. 40(10), 2321-2334.

Baloff, N. (1970). Startup Management. IEEE Transactions on Engineering Management, 17,132-141.

Basu, R., Basu, A., \& Nair, K. (2002). Muscle changes in aging. The Journal of Nutrition, Health and Aging, Vol. 6(5), 336-341.

Belbase, A., \& Sanzenbacher, G. T. (2016). Cognitive aging and Ability to Work. Boston: Center for Retirement Research.

Bellew, J. W., Symons, B. T., \& Vandervoort, A. A. (2005). Geriatric fitness: effects of aging and recommendations for exercise in older adults. Cardiopulmonary Physical Therapy Journal (American physical therapy association, cardiopulmonary section), Vol. 16(1), 20-31.

Berg, J. V., \& Neely, G. (2006). Performance on a simple reaction time task while sleep deprived. Perceptual and Motor Skills, 589-599.

Boenzi, F., Digiesi, S., Mummolo, G., \& Romano, V. A. (2015). Modelling workforce aging in job rotation problems. IFAC-PapersOnline, Vol. 48(3), 604-609.

Boudreau, J., Hopp, W., McClain, J. O., \& Thomas, L. J. (2003). On the Interface Between Operations and Human. Manufacturing \& Service Operations Management, Vol. 5(3), 179-202.

Bridger, R. S. (2009). Introduction to Ergonomics (3rd ed.). United state of America: CRC Press.

Broadbent, D. E. (1971). Decision and stress. London: Academy Press Inc.

Buckley, J. J. (1985). Fuzzy hierarchical analysis. Fuzzy Sets and Systems, Vol. 17, 233-245.

Chaffin, D. G. (2006). Occupational Biomechnics (4th ed.). New Jersey: John Wiley.

Chang, D.-Y. (1996). Theory and methodology: application of extend analysis method on fuzzy AHP. European Journal of Operation Research, Vol. 96, 649-655. 
Chen, C.-T. (2001). A fuzzy approach to select the location of the distribution center. Fuzzy Sets and Systems, Vol. 118, 65-73.

Chou, S.-Y., Chang, Y.-H., \& Shen, C.-Y. (2008). A fuzzy simple additive weighting system under group decision making for facility selection with objective/subjective attributes. European Journal of Operation Research, Vol. 189, 132-145.

Clark, A., \& Oswald, A. (1996). Is job satisfaction U-shape in age? Journal of Occupational and Organisational Psychology, Vol. 69, 57-81.

Crawford, O. J., Graveling, R. A., Cowie, H., Dixon, K., \& MacCalman, L. (2010). The health, safety and health promotion needs of the older workers . Leicester shire: IOSH.

Deary, I. J., Der, G., \& Ford, G. (2001). Reaction times and intelligence differences a population based cohort study. Intelligence, 389-399.

Der, G., \& Deary, I. J. (2006). Age and sex differences in reaction time adulthood: result from the united kingdom health and life style survey. Psychology and Aging, Vol. 21 (1) 62-73.

Digiesi, S., Kock, A. A., Mummolo, G., \& Rhoda, J. E. (2006). The effect of dynamic worker behavior on flow line performance. IFAC proceeding, Vol. 39(3), 341-346.

Dode, P., Greig, M., Zolfaghari, S., \& Neumann, W. P. (2017). Integrating human factors into discrete event simulation: a proactive approach to. International Journal of Production Research,, Vol. 54(10),3105-3117.

Doroudgar, S., Chuang, H. M., Perry, P. J., Thomas, K., Bohnert, K., \& Canedo, J. (2017). Driving performance comparing older Vs younger drivers. Traffic Injury Prevention, Vol.18(1), 41-46.

Drabe, D., Hauff, S., \& Richter, N. F. (2015). Job satisfaction in aging work force: an analysis of the USA, Japan and Germany. International Journal Human Resources Management, Vol. 26(6), 783-805.

Ellis, R. D., Pines, E., \& Allaire, J. C. (1999). Performance implications of older workers in technological manufacturing environments: A task-analysis/human reliability perspective. International Journal of Computer Integrated Manufacturing, Vol. 12(2), 104-112.

Falck, A. C., \& Rosenqvist, M. (2012a). What are the obstacles and needs of proactive ergonomics measures at early product development stages?-an interview in five Sweden companies. International Journal of Industrial Ergonomics, Vol. 42, 406-415.

Fan, Z.-P., Hu, G.-F., \& Xiao, S.-H. (2004). A method for multi attribute decision making with the fuzzy preference relationship. Computers \& Industrial Engineering, 321-327.

Fleg, J. L., Morrell, C. H., Bos, A. G., Brant, L. J., Talbot, L. A., Wright, J. G., \& Lakatta, E. G. (2005). Accelerated longitudinal decline of aerobic capacity in healthy adults. Circulation, Vol. 112(5), 674-682.

Galen, L. (1987). Applied Ergonomics. Oxford: Butterworth.

Gall, B., \& Parkhouse, W. (2004). Physical capacity as a function of age in heavy manual work. Ergonomics, Vol. 47(6), 671-687.

Giniger, S., Dispenzieri, A., \& Eisenberg, J. (1983). Age, experience, and performance on speed and skill jobs in an applied setting. Journal of Applied Psychology, Vol. 68(3), 469-475. 
Govindaraju, M., Pennathur, A., \& Mital, A. (2001). Quality improvement in manufacturing through human performance enhancement. Integrated Manufacturing Systems, 360-367.

Hawkins, S. A., \& Wiswel, R. A. (2003). Rate and Mechanism of Maximal oxygen consumption decline with aging implications for exercise training. Sports Medicine, Vol. 33(12), 877-888.

Hunter, J. E. (1986). Cognitive ability,cognitive aptitudes, job knowledge, and job performance. Journal of Vocational Behavior, 389-399.

Hunter, J. E., \& Schmidt, F. L. (1996). Intelligence and job performance: economic and social implications. Psychology, Public Policy, and Law, Vol. 2(3-4), 447-472.

Ilmarinen, J. (2002). Physical requirement associated with the work of aging workers in the European Union. Experimental Aging Research, Vol. 28, 7-23.

Isik, Z., \& Aladag, H. (2017). A fuzzy model to asses sustainable performance of the construction industry from urban regeneration perspective. Journal of Civil Engineering and Management, Vol. 23 (4), 499-509.

Jaber, M. Y. (2001). Learning curves theory, models and applications. London: CRC Press.

Jaber, M. Y., \& Bonney, M. (1996). Production Breaks and the Learning Curve : The Forgetting phenomenon. Applied Mathematical Modelling, vol. 20, 161 - 169.

Jaber, M. Y., \& Bonney, M. (1997). Comparative Study of Learning Curve With Forgetting. Journal of Mathematical Modelling, vol. 21, 523 - 531.

Kahraman, C., Ertay, T., \& Buyukozkan, G. (2006). A fuzzy optimization model for QFD planning process using analytical network approach. European Journal of Operation Research, VOL. 171, 390-411.

Kenny, G. P., Groeller, H., McGinn, R., \& Flouris, A. D. (2015). Age, human performance, and physical employment standards. Applied Physiology Nutrition and Metabolism, Vol. 41(6), 92-107.

Kowalski-Trakofler, K., Steiner, L. J., \& Schwerha, D. J. (2005). Safety consideration for the aging workforce. Safety Science, Vol. 43, 779-793.

Kumar, T. V. (2017). Factors impacting job satisfaction among police personal in India: A multidimensional analysis. Georgia : International Criminal Justice Review.

Kwong, C., \& Bai, H. (2003). Determining the importance weights for consumer requirements in QFD using Fuzzy AHP with extend analysis approach. IIE Transactions, Vol. 35, 619-626.

Laarhoven, P., \& Pedrycz, N. (1983). A fuzzy extension of saaty's priority thoery. Fuzzy Sets and Systems, Vol.11(3), 229-241.

Lamkull, D., Hanson, L., \& Ortengren, R. (2009). A comparative study of digital human modelling simulation result and their outcomes in reality: a case study within manual assembly of automobiles. International Journal of Industrial Ergonomics, Vol. 39, 428-441.

Leyk, D., Ruther, T., Wunderlich, M., Sievert, A., Dieter, e., Witzki, A., Lollgen, H. (2010). Physical performance in middle and old age: good news for our sedentary and aging society. Deutsches Arzteblatt, Vol. 107(46), 809. 
Likert, R. (1932). A technique for the measurement of attitudes. Archives of psychology , Vol. 55, 22140.

IImarinen, J. E. (2001). Aging workers. Occupational and Environmental Medicine, Vol. 58(8),546-552.

McDaniel, M. A., Hunter, J. E., \& Scmidt, F. (1988). Job experience correlates job performance. Journal of Applied Psychology, Vol.73(2), 327-330.

McDaniel, M. E., Schmidt, F. L., \& Hunter, J. E. (1988). Job experience correlates of job performance. Journal of Applied Psychology, Vol.73(2), 327-330.

McEvoy, G. M., \& Cascio, W. F. (1989). Cumulative evidence of the relationship between employee age and job performance. Journal of Applied Psychology, Vol. 74(1), 11-17.

Mendonca, G. V., Pezarat-Correia, P., Vaz, J. R., Silver, L., \& Heffernan, K. S. (2017). Impact of aging on endurance and neuro-muscular physical performance: the role of vascular senescence. Sports Med , Vol. 47, 583 - 598.

Mikhailov, L. (2000). A fuzzy programming method for driving priorities in the analytic hierarchy process. Fuzzy Sets and Systems, Vol. 134(3), 365-385.

Mikhailov, L. (2003). Deriving priorities from fuzzy pairwise comparison. Fuzzy Sets and Systems, Vol. 134, 365-385.

Moskaliuk, J., Burmeister, C. P., Landkammer, F., \& Renner, B. (2017). Environmental effects on cognitive and decision making of knowledge workers. Journal of Environmental Psychology, Vol. 49, 43-54.

Narahari, C. L., \& Koneru, D. K. (2017). A study on the role of occupational stress in organisations. International Journal of Engineering Technology, Engineering, Management and Applied Sciences, Vol.5(1), 53-59.

Nebmhard, D. A., \& Osothsilp, N. (2002). Task Complexity Effect on Between Individual Learning / Forgetting Variability. International Journal Of Industrial Ergonomics, vol. 29, 297 - 306.

Neumann, W. P., \& Medbo, P. (2017). Simulation operator learning during production ramp-up in parallel vs serial flow production. International Journal of Production Research, Vol. 55(3), 845-857.

Oborne, D. J. (1995). Ergonomics at Work (3rd Edition). West Sussex: John Wiley.

Palmer, K. T., Harris, E. C., \& Coggon, D. (2006). Capal Tunnel Syndrome and It's Relation To Occupation: A Systematic Literature Review. Oxford Journal Of Occupational Medicine, vol.57(1), $57-66$.

Peruzzini, M., \& Pellicciari, M. (2017). A framework to design a human-centered adaptive manufacturing system. Advanced Engineering Informatics, 1-20.

Reagans, R., Argote, L., \& Brooks, D. (2005). Individual Experienced and Inexperience Working Together: Predicting learning rate from knowing who knows what and knowing how to work together. Management Science, vol.51(6), 869 - 881.

Rhodes, S. R. (1983). Age-related differences in work attitudes and behavior. A review and conceptual analysis. Psychology Bulletin, Vol. 93(21), 328-367. 
Robertson, A., \& Tracy, C. S. (1998). Health and productivity of older workers. Scand. J. Work Environ. Health, Vol. 24(2), 85-97.

Rodriguez, D., Buyens, D., \& Landeghem, H. V. (2016). Impact of lean production on perceived job autonomy and job satisfaction: an experimental study. Human Factors and Ergonomics in Manufacturing \& Service Industries, 159-179.

Ross, T. J. (1995). Fuzzy Logic with Engineering Applications. New York: McGraw-Hill.

Saaty, R. W. (1987). The analytical hierarchy process-what it is and how it is used. Mathematical Modelling, Vol. 9, (3-5), 161-176.

Saaty, T. L. (2008). Decision making with the analytical hierarchy process. Int. J. Services Sciences, Vol. 1(1), 83-98.

Salthouse, T. A. (1996). Implication of adult age differences in cognition for work performance. International Scientific Conference (pp. 15-28). Stockholm: National institute for working life.

Salvia, E., Petit, C., Champely, S., Rienzo, F. D., \& Collect, C. (2016). Effects of age and task on drivers response accuracy and reaction time when responding to traffic lights. Frontiers in Aging

Savinainen, M. (2004a). Physical Capacity and Workload Among Aging Workers. Tempere: Medical school university of Tempere.

Savinainen, M., Clas-Hakan, N., Olli, K., \& IIIlmarine, J. (2004b). Changes in Physical Capacity Among Middle-Aged municipal employee over 16 years. Experimental Aging, Vol. 30(1), 1-22.

Schibye, B., \& Hansen, A. F. (2001). Aerobic power and muscle strength among young and elderly workers with and without physical demanding work tasks. Applied Ergonomics, Vol. 32, 425431.

Schmid, B. (2005). Human factors in complex systems the modelling of human behavior. Simulation in wider Europe, 19th Conference on Modelling and Simulation, 5-14.

Schmidt, L. F., Outerbridge, A. N., \& Hunter, J. E. (1986). Impact of experience and ability on job knowledge, work sample performance, and supervisory ratings of performance. Journal of Applied Psychology, Vol.71(30) 32-439.

Scwab, D. P., \& Heneman, H. G. ( 1976). Effect of age and experience on productivity. Academy of management proceedings, Vol. 1976(1), 281-283.

Sehl, M. E., \& Yates, F. E. (2001). Kinetics of human aging: I. rates of senescence between age 30 and 70 years in healthy people . Journal of Gerontology, Vol. 56A(5), B198-B208.

Shafer, M. S., Nembhard, A. D., \& Uzumeri, V. M. (2001). The effect of learning, forgetting, and heterogeneity on assembly line productivity. Management Science, 47 (12), 1639-1653.

Shephard, R. J. (2000). Aging and productivity: some physiological issues. International Journal of Industrial Ergonomics, Vol. 25, 535-545.

Skirbekk, V. (2008). Age and productivity capacity : Descriptions, causes and policy options. Ageing Horizons, 4-12.

Stanley R., P. (1985). Adult Age Differences in Short Term Forgetting. International Journal of Psychonomics vol. 60(1), 83 - 101. 
Stead, M., \& Page, M. (1983). Ergonomics At Work. England: Manpower Service Commission.

Svetina, M. (2016). The reaction times of drivers aged 20-80 during a divided attention driving. Traffic Injury Prevention, Vol. 17(8), 810-814.

Tolga, E., Demirlan, M. L., \& Kahraman, C. (2005). Operation system selection using fuzzy replacement analysis and analytical hierarchy process. International Journal of Production Economics , Vol. 97, 89-117.

Tseng, W.-J., Ding, J.-F., \& Chang, K.-H. (2017). Evaluating key eviromental risk factors for pollution at international port in Taiwan. Brodogradnja, Vol. 68(1), 1-15.

Tzeng, G.-H., \& Huang, J.-J. (2011). Multiple attribute decision making: Methods and applications. USA: CRC Press.

Visser, I., Raijmakers, M. E., \& Molenaar, P. C. (2007). Characterizing sequence knowledge using online measures and hidden Markov models. Memory \& Cognition, Vol. 35(6), 1502-1517.

Waldman, D. A., \& Avolio, B. J. (1986). A meta-analysis of age difference in job performance. Journal of Applied Psychology, Vol. 71(1),33-38.

Wang, Q., \& Chatwin, C. R. (2005). Key issues and developments in modelling and simulation-based technologies for manufacturing system analysis, design and performance evaluation. International Journal of Advanced Manufacturing Technology, Vol. 25, 1254-1265.

Wang, Q., Lassalle, S., A., R. M., \& Owen, G. W. (2009). Analysis of a Linear Walking Worker Line Using a Combination Of Computer Simulation and Mathematical Modeling approaches. Journal of Manufacturing Systems, vol. 28, 64 - 70.

Wang, Q., Owen, G. w., \& Mileham, A. R. (2007). Determining numbers of workstations and operators for linear walking-worker assembly line. International Journal of Computer Integrated Manufacturing , Vol. 20(1), 1-10.

Wang, Q., Sowden, M., \& Mileham, A. R. (2012). Modelling human performance withing an automotive engine assembly line. International Journal of Advanced Manufacturing Technology vol. 68(1-4), 141-148.

Warr, P. (1994). Work and Aging: a European perspective. Great Britain: Tailor and Francis Ltd.

Welford, A. (1968). Fundamentals of skill. London: Butler and Tunner Ltd.

Wirojanagud, P., Gel, E. S., Fowler, J. W., \& Cardy, R. (2007). Modelling inherent worker differences for workforce planning. International Journal of Production Research, Vol 45(3), 525-553.

Woodson, W., Tillman, E., \& Tillman, B. (1992). Human factors design handbook: information and guidelines for the design of systems, facilities, equipment, and products for human use. In W. E. Woodson, B. Tillman, \& B. Tillman, Reaction time (pp. 630-631). USA: McGraw-Hill.

Yuksel, I., \& Dagdeviren, M. (2008). Developing a fuzzy analytical process (AHP) model for behavior based safety management. Information Sciences, Vol. 178,, 1717-1733.

Zhu, K.-J., Jing, Y., \& Chang, D.-Y. (1999). A discussion on extent analysis method and application of fuzzy AHP. European Journal of Operation Research, Vol. 116, 450-456. 
Zwick, T., \& Gobel, C. (2009). Age and productivity-evidence from link employee data. Zew-center for European Economic Research discussion paper (09-020). 